\title{
MICCAI 2014 special issue
}

\author{
Nobuhiko Hata $^{1}$ • Joachim Hornegger ${ }^{2}$
}

Published online: 26 July 2015

(c) CARS 2015

In 2014, the annual meeting of Medical Image Computing and Computer-Assisted Intervention (MICCAI) was held from September 14 to 18 in Boston, USA. This was the seventeenth annual meeting for MICCAI, but only the second time it was held in Boston since MICCAI community met there in 1996. As before, the meeting was held on sunny days at the Massachusetts Institute of Technology campus. The conference was characterized by vibrant and constructive discussions on biomedical image computing, computer-assisted interventions, and medical robotics. In total, we invited 253 poster presentations selected from 862 paper submissions following a rigorous review procedure by 52 experts in the field.

The current special issue of the International Journal of Computer Assisted Radiology and Surgery (IJCARS) includes five research papers nominated from this selective list of excellent papers presented at the MICCAI meeting. These papers present contemporary findings in fields shared by both MICCAI and the IJCARS journal. In fact, the current

Nobuhiko Hata

hata@bwh.harvard.edu

Joachim Hornegger

joachim.hornegger@fau.de

1 Department of Radiology, Brigham and Women's Hospital, L1-050, 75 Francis St., Boston, MA 02115, USA

2 Pattern Recognition Lab, Friedrich-Alexander-Universität Erlangen-Nürnberg, Martensstrasse 3, 91058 Erlangen, Germany issue is the first IJCARS special issue to highlight papers from MICCAI, following a new arrangement between the MICCAI society and the IJCARS editorial office. We are delighted to present this first special issue on MICCAI, which extends the breadth of the IJCARS journal.

In this issue, we highlight papers describing the enhancement of image-guided therapy with advanced computing. For instance, we are fortunate to publish papers by Zuluaga et al. and Coevoet et al., which present image processing methods to enhance stereoscopic EEG implantation in neurosurgery and radiotherapy, respectively. The paper by Billings et al. describes an extension to the popular Iterative Closest Point algorithm, which may enormously enhance the accuracy and versatility of image-to-image and image-to-patient registration in image-guided therapies. Also in this issue, Maier-Hein et al. address interventions in the context of annotating endoscopic images. The paper by Rodas et al. addresses image-guided therapy from the safety perspective for medical staff, by making a radiation risk map for the intervention room. All of these exciting papers warrant journal publication, but not necessarily in traditional journals that specialize only in either biomedical image analysis or therapy.

Upon the invitation for submissions to this issue, we mandated that the authors of these papers extend their corresponding MICCAI papers and then once again undergo a rigorous regular IJCARS review process. Considering that MICCAI 2014 had only a $29 \%$ acceptance rate and that the MICCAI papers by themselves were a product of significant work, the papers accepted and presented in this special issue have tremendous content value and potential impact for our community.

Lastly, the special issue editors thank the reviewers of these papers and the staff in the editorial office of IJCARS for their contributions in bringing these papers to the public. 